\title{
Processo de trabalho e riscos para a saúde dos trabalhadores em uma indústria de cimento
}

Fátima Sueli Neto Ribeiro 1

Simone Oliveira 2

Marcelo Moreno dos Reis 3

Célia Regina Sousa da Silva 4

Marco Antônio Carneiro Menezes 2

Ana Elisa Xavier de Oliveira e Dias 2

Josino Costa Moreira 2

Gisele Sayuri Kuryiama 2

\author{
The work process and occupational \\ health risks in a cement factory
}

1 Programa de Saúde do Trabalhador, Secretaria de Estado de Saúde. Rua México 128, 4o andar, Rio de Janeiro, $R J$ 20031-142, Brasil.

2 Centro de Estudos da Saúde do Trabalhador e Ecologia Humana, Escola Nacional de Saúde Pública, Fundação Oswaldo Cruz. Rua Leopoldo Bulhões 1480, Rio de Janeiro, $R J$ 21040-210, Brasil. 3 Secretaria Municipal de Saúde de Volta Redonda. Rua 566 31, Volta Redonda, RJ 27091-390, Brasil.

4 Departamento de Físico-Química, Instituto de Química, Universidade Federal do Rio de Janeiro. Centro de Tecnologia, Bloco A, sala 408 Cidade Universitária, Rio de Janeiro, $R J$ 21949-900, Brasil.

\begin{abstract}
The authors evaluate the work process and its effect on workers' health in a cement factory in the State of Rio de Janeiro. The interactive methodology consisted of different approaches to assessing the workplace through the incorporation of various institutions working in the field of Workers' Health, professionals from different backgrounds, and the trade union, valorizing the workers' experience and actively contributing to the surveillance process under the Unified National Health System (SUS). Levels of particulate matter and noise were measured. The mean level of free crystalline silica in the particulate matter was $2 \%$, resulting in a tolerance limit as specified under Brazilian legislation (NR-15), or 2.0mg/m3. The concentration of particles both in samples collected in the workers' respiratory zone and in area samples varied from 3.59 to $52.44 \mathrm{mg} / \mathrm{m}^{3}$. Noise varied from $83 \mathrm{~dB}$ to $110 \mathrm{~dB}$. The majority of the values were higher than the maximum limits set by Brazilian legislation. These results, together with the opinions expressed by the workers themselves, showed an unhealthy workplace and work process, placing the workers' health at risk.
\end{abstract}

Key words Ocupational Health; Particles; Cement Industry; Occupational Noise

Resumo A avaliação do processo de trabalho sobre a saúde de trabalhadores de uma fábrica de cimento, localizada no Estado do Rio de Janeiro, é relatada. A metodologia interativa utilizada, constou de distintas formas de avaliação do ambiente de trabalho através da incorporação de várias instituições, com atribuição na área de Saúde do Trabalhador, de técnicos de diversas formações, do sindicato e da valorização da experiência do trabalhador, contribuindo ativamente no processo de vigilância do SUS. Os níveis de material particulado e de ruído foram medidos. $O$ porcentual médio de sílica livre cristalina encontrado no material particulado, foi de 2\%, o que resultou em um limite de tolerância, determinado como especificado na Legislação Brasileira (NR-15), de 2,0mg/m3. A concentração de partículas, tanto em amostras coletadas em nível da zona respiratória dos trabalhadores, quanto às amostras de área, variou de 3,59 a 52,44mg/ $\mathrm{m}^{3}$, o nível de ruído situou-se entre $83 \mathrm{~dB}$ e $110 \mathrm{~dB}$. A maioria dos valores encontrados superam o valor limite estabelecido pela Legislação Brasileira. Esses resultados, somados ao registro do olhar dos trabalhadores, revelaram um ambiente e processo de trabalho insalubre, colocando em risco a saúde dos operários.

Palavras-chave Saúde Ocupacional; Material Particulado; Indústria do Cimento; Ruído Ocupacional 


\section{Introdução}

A introdução de inovações tecnológicas e organizacionais nos processos de trabalho, têm trazido uma maior complexidade às relações produtivas, bem como ao seu entendimento. No Brasil, a heterogeneidade dos processos de mudança, observada nos vários setores industriais nos últimos anos e, às vezes, dentro de uma mesma unidade industrial, exige para o seu entendimento, a adoção de uma abordagem multidisciplinar adequada à esta realidade.

Tradicionalmente, os estudos realizados para a compreensão da relação processo de trabalho e saúde, se fundamentam em práticas convencionais da Medicina do Trabalho e da Engenharia de Segurança, onde o entendimento dessa relação é resultante exclusiva da ação isolada de agentes patogênicos sobre o corpo do trabalhador ou, no máximo, pela multiplicidade e interação de grupos de agentes em que a ênfase é voltada para a proteção "contra" os riscos (Vasconcellos, 1994).

Essa abordagem limitada encobre os conflitos das relações sociais existentes nos processos de trabalho. A apreensão dessa dinâmica social é fundamental para um enfoque global do processo de trabalho e o processo de desgaste dele decorrente, que impõem aos trabalhadores uma série de riscos provenientes das relações sociais de produção, Tambellini (1988).

Neste contexto, investigar a saúde dos trabalhadores é condição sine qua non para a construção de um novo modo de entendimento e análise do binômio saúde-doença coletiva enquanto processo social (Laurell \& Noriega, 1989)

É a partir dessa perspectiva, que o trabalho de Vigilância em Saúde do Trabalhador deve utilizar metodologias interativas capazes de identificar e compreender os problemas de saúde dos trabalhadores, bem como o desenvolvimento e a implementação de ações que objetivem a transformação dos ambientes insalubres e perigosos de trabalho. Em geral, o entendimento desses problemas exige uma articulação complexa, envolvendo conhecimentos interdisciplinares e que não pode desprezar o saber operário, sistematizado a partir do registro do olhar dos trabalhadores sobre seu ambiente e sua percepção de adoecimento, de risco e de acidentes. Assim, é necessário construir-se uma metodologia de intervenção interativa, que conte com a participação dos trabalhadores em todas as suas etapas (Vasconcelos \& Ribeiro, 1997).

A indústria de produção de cimento é potencialmente, uma das mais preocupantes quanto à possibilidade de exposição de trabalhadores a material particulado devido ao fato de trabalharem com material sólido, onde a possibilidade de geração de poeiras é elevada, expondo o trabalhador a riscos. Outra característica da exposição ao cimento, é que não restringe-se aos muros das fábricas, atingindo especialmente os trabalhadores da construção civil, e também o fato de que, dependendo do processo produtivo, outras substâncias como por exemplo, metais, podem estar presente no produto final, como destaca o relatório técnico sobre as indústrias cimenteiras de Cantagalo (Rio de Janeiro), realizado por uma equipe do Centro de Estudos da Saúde do Trabalhador e Ecologia Humana (CESTEH, 2000). No entanto, pouco se conhece da realidade das indústrias brasileiras de cimento, pois, é pequeno o número de estudos disponíveis na literatura (Santos, 1997). Alguns estudos internacionais, indicam uma alta correlação entre o nível de exposição ao material particulado e doenças respiratórias dos trabalhadores (Alvear-galindo, 1999; Vestbo, 1990; Yang, 1996).

\section{Material e métodos}

No intuito de contemplar as muitas questões presentes no trabalho, as ações de Vigilância, desenvolvidas pelo Sistema Único de Saúde SUS/Rio de Janeiro, vêm construindo uma metodologia de intervenção interativa. Tal intervenção, sob o horizonte da mudança tecnológica e/ou da reorganização do processo de trabalho, busca dar conta dos princípios de ações interdisciplinares e pluriinstitucionais, contando com a participação da representação dos trabalhadores em todas as etapas (Ribeiro, 1996).

A ação da vigilância se inicia a partir de uma denúncia do Sindicato dos Trabalhadores da Indústria da Construção Civil de Volta Redonda/Rio de Janeiro, com relação às condições nocivas de trabalho na indústria de cimento. Para a realização da ação, através do Conselho Estadual de Saúde do Trabalhador do Rio de Janeiro (CONSEST), associaram-se aos Programas de Saúde do Trabalhador (PSTs) o CESTEH/Fundação Oswaldo Cruz (FIOCRUZ), a Faculdade de Psicologia da Universidade Federal Fluminense (UFF), a Comissão Estadual de Pneumopatia Ocupacional do Rio de Janeiro e a Universidade Federal do Rio de Janeiro.

A fábrica de cimento estudada, localiza-se numa área densamente povoada do município de Volta Redonda, região do Médio Paraíba do Estado do Rio de Janeiro, conta com 134 trabalhadores, sendo que 38 trabalham na adminis- 
tração, incluindo 4 mulheres, 29 na manutenção e 67 na produção.

A ação de vigilância efetivou-se em três momentos, a saber:

1) O primeiro momento, consistiu no reconhecimento técnico das condições de trabalho e de controle da saúde dos trabalhadores, realizados pela empresa, através de inspeções técnicas mensais, realizadas pelos técnicos da Secretaria de Estado de Saúde (SES) e da Secretaria Municipal de Saúde (SMS), pelas instituições que acompanham a Câmara Técnica da Construção Civil do CONSEST, o CESTEH/FIOCRUZ, além de diversas assessorias incorporadas em várias fases da avaliação, tais como a Comissão Estadual de Pneumopatia Ocupacional e a Faculdade de Psicologia da UFF. Em todos os momentos o sindicato participou do processo. Para o registro das informações foi utilizado, além dos relatórios individuais, as informações prestadas pela empresa por meio de preenchimento do documento Declaração do Processo de Produção Relacionado às Questões de Saúde e Meio Ambiente (DEPRO), instrumento de registro de dados epidemiológicos utilizado pela SES/Rio de Janeiro.

2) Mensuração dos riscos, através da determinação da concentração de poeira mineral presente no ambiente de trabalho e pela determinação do nível de ruído. Foram empregadas as metodologias recomendadas pelo Ministério do Trabalho do Japão, (Japan Association for Working Environment Measurement, 1991) e pelo Ministério do Trabalho do Brasil, (Ministério do Trabalho, 1996). Duas formas de amostragem foram realizadas para a avaliação da concentração de poeira mineral: a amostragem individual (Soto et al., 1990), feita com Airchek Sampler model 224-PCXR4 da SKC com fluxo constante de 1,9 L/min e a amostragem de área adotada pela Japan Industrial and Health Association - JISHA (1985). Nesse caso, foram empregados três sistemas diferentes: (1) coleta realizada com bomba Sibata, modelo IP-20T, com fluxo constante de $15 \mathrm{~L} / \mathrm{min}$, acoplada a um seletor do tamanho das partículas do tipo multi-estágios (amostrador HE-horizontal elutriator), que permite a coleta de poeira respirável sobre papel de filtro T60A20; (2) coleta feita com Bomba Sibata, modelo IP-20T, com fluxo constante de $20 \mathrm{~L} / \mathrm{min}$, acoplada a um amostrador de poeira Roken TR (TR-Total and Respirable), no qual a poeira respirável é coletada sobre papel de filtro T60A20 e a não respirável sobre uma placa de acrílico recoberta com silicone (Kimura, 1978a, 1978b); (3) Contador digital de partículas Sibata, modelo P-5H (Dust Indicator P-5H), para avaliação do nível de poei- ra respirável, que é expresso em número de contagens por minuto.

Para a determinação do nível de ruído utilizou-se um medidor do nível de som modelo La-220S (ONO SOKKI).

A determinação da massa de poeira mineral coletada em cada papel de filtro foi feita por gravimetria. Dividindo-se a massa encontrada pelo volume de ar amostrado, foi obtida a densidade de massa de poeira, expressa em $\mathrm{mg} / \mathrm{m}^{3}$. As amostras coletadas também foram analisadas pela técnica de difração de raios-X, permitindo assim, que o porcentual de sílica livre cristalina contido na poeira fosse quantificado.

3) Registro da percepção dos trabalhadores a cerca das condições, do processo de trabalho e dos riscos dele decorrentes. Esta etapa foi feita através da aplicação de um questionário, contendo tanto questões que envolviam respostas diretas (questões fechadas), quanto questões abertas que permitiam uma maior flexibilidade nas respostas e na análise dos dados coletados.

Essa etapa do processo de vigilância é fundamental, pois além da incorporação da perspectiva dos trabalhadores, fornece subsídios para um diagnóstico situacional da organização do trabalho e das condições ambientais da empresa. O questionário foi aplicado em 66 trabalhadores, destes $75 \%$ funcionários da Empresa de Cimento e $25 \%$ de empresas contratadas por esta (empreiteiras).

A amostra utilizada para fins de aplicação do questionário, não se prendeu a parâmetros de seleção estatística, mas visou registrar o máximo de impressões no dia em que foi aplicado, contando com a relação de confiança, já conquistada pelas sucessivas visitas da equipe de inspeção, e incluiu os trabalhadores dos três turnos.

\section{Resultados}

A interpretação dos resultados, foi feita concomitantemente com o entendimento geral do processo de produção utilizado por essa empresa, cuja organização é mostrada, em linhas gerais, na Figura 1.

Além do calcário e da argila, materiais básicos utilizados na produção de cimento, esta empresa utiliza também no seu processo de fabricação, a escória siderúrgica e o gesso.

O gesso é adicionado à mistura pois atua como regulador do tempo de endurecimento. A escória adicionada ao cimento, serve para diminuir o calor de hidratação e confere maior resistência. Esta peculiaridade difere a empre- 
Figura 1

Diagrama esquemático do processo de produção de cimento utilizado em Volta Redonda, Rio de Janeiro.

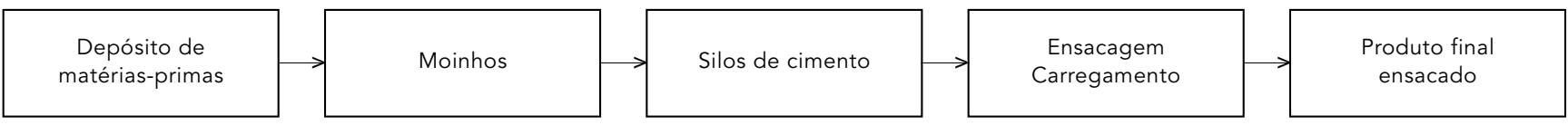

sa estudada das demais indústrias típicas deste setor.

As matérias primas são transportadas através de uma ponte rolante, dos galpões de depósito até o local onde é feita a moagem. Esta ponte é dotada de comandos elétricos e operada por quatro operários, que trabalham em turnos diferenciados de seis horas diárias. Devido à sua condição precária, os trabalhadores que a operam ficam expostos constantemente, a vários fatores de risco à saúde, tais como por exemplo, a alta concentração de partículas em suspensão (poeiras) devido à falta de isolamento da cabine de operação; falta de equipamentos de proteção, segurança e comunicação. Além disso, este trabalho é monótono e solitário. Do total de trabalhadores entrevistados, $20 \%$ considerou ser este um dos locais de trabalho mais perigosos em toda a fábrica.

A pesagem e a mistura dos componentes são feitas nas balanças dosadoras. Em seguida, essa mistura é transportada para os moinhos de cimento, que são moinhos de bolas rotativos, que funcionam em circuito fechado e com granulometria controlada por um separador.

Os silos de cimento são os depósitos do produto final da moagem, antes do ensacamento. De acordo com a opinião de $11 \%$ dos trabalhadores entrevistados, estes silos são os locais que mais oferecem riscos à integridade física dos operários, devido à altura dos mesmos.

O ensacamento é feito por três ensacadeiras rotativas com capacidade variando de 1.600 a 1.800 sacos/hora. O cimento é transportado dos silos através de um sistema mecânico, até as ensacadeiras, onde é injetado nos sacos por meio de bicos. Os sacos de papel são colocados manualmente nos bicos das ensacadeiras por um operador, posicionado em frente ao equipamento. Essa tarefa é realizada em sistema de revezamento por dois operadores, em períodos de uma hora.

Depois de ensacado, o cimento é transportado por esteiras rolantes até as mesas móveis transportadoras, onde é realizada a operação de carregamento dos caminhões transportadores. Essa operação é executada por dois trabalhadores, sendo um responsável pelo controle das mesas transportadoras e o outro pelo ajuste dos sacos nas carrocerias. O operador ajusta o saco de cimento apoiando-o com a coxa e deixando-o tombar pela ação da gravidade, formando assim, pilhas nos caminhões. Alcança-se uma média diária de carregamento de 120 a 130 caminhões com 300 a 500 sacos de cimento cada.

\section{Avaliação ambiental}

De acordo com os resultados obtidos pela análise por difração de raios-X, o porcentual médio de sílica livre cristalina $\left(\mathrm{SiO}_{2}\right)$ encontrado nas amostras coletadas, foi de $2 \%$. Para este tipo de exposição, não é adotado um valor fixo para o limite de tolerância (LT), pois este valor depende do porcentual de $\mathrm{SiO}_{2}$ presente no material particulado. Conforme estabelecido na Norma Regulamentadora 15, anexo 12 do Ministério do Trabalho do Brasil, o valor de LT pode ser calculado empregando-se a Equação 1.

$$
L T\left(m g / m^{3}\right)=\frac{8,0}{\% S i O_{2}+2}
$$

(Equação 1)

Desse modo, com base na Legislação Brasileira, o valor de $2,0 \mathrm{mg} / \mathrm{m}^{3}$ foi adotado como limite de tolerância para a exposição ocupacional à poeira mineral respirável, contendo $2 \%$ de sílica livre cristalina.

De acordo com a metodologia de cálculo adotada pela JISHA, a concentração média de poeira, contendo $2 \%$ de sílica livre cristalina permitida em um ambiente de trabalho fechado, seria de $2,01 \mathrm{mg} / \mathrm{m}^{3}$.

Os resultados das avaliações individuais são mostrados na Tabela 1.

Todos os valores encontrados nestas amostragens pessoais, foram superiores àquele re- 
comendado como limite de tolerância pela legislação brasileira $\left(2,0 \mathrm{mg} / \mathrm{m}^{3}\right)$.

A média geométrica dos valores obtidos nos trabalhadores ECC1 a ECC3 (ensacamento fechado), foi de $21,63 \mathrm{mg} / \mathrm{m}^{3}$ com um desvio padrão de 3,83. A exposição medida no assistente de ensacamento foi inferior à dos ensacadores, devido à sua maior mobilidade.

No caso de ensacamento aberto (amostras ECC4 e ECC5), a média geométrica foi de 10,76 $\mathrm{mg} / \mathrm{m}^{3}$ e o desvio padrão de $1,12 \mathrm{mg} / \mathrm{m}^{3}$.

Mesmo a amostragem realizada no carregador, embora menos exposto à poeira, apresentou valor da concentração de material particulado de $3,59 \mathrm{mg} / \mathrm{m}^{3}$, ainda superior ao limite recomendado pela legislação.

Os valores das concentrações de material particulado encontrado em três setores, são apresentados na Tabela 2.

A média geométrica dos valores das concentrações encontradas no setor de ensacamento fechado, é de $4,81 \mathrm{mg} / \mathrm{m}^{3}(\sigma=1,90)$, enquanto que no setor de ensacamento aberto, este valor foi de $1,9 \mathrm{mg} / \mathrm{m}^{3}(\sigma=6,42)$. Essa grande variação observada na concentração de sílica neste ambiente, é devida ao fato de se tratar de uma área coberta mas aberta lateralmente.

Utilizando a metodologia proposta pela JISHA, para classificação dos locais de trabalho, os ambientes monitorados são classificados como classe III, o que significa dizer que são ambientes com elevado grau de contaminação.

Os níveis de emissão, observados durante a operação de carregamento, variaram de 0,24 a $10,85 \mathrm{mg} / \mathrm{m}^{3}$. A grande variação verificada pode ser explicada pela influência da direção do vento no ponto amostrado, uma vez que o caminhão de transporte fica estacionado em uma área aberta. Esses valores mostram que o nível de exposição ainda é elevado, mesmo em se tratando de área externa, não coberta.
Além da poeira abundante, sobressai a qualquer observador, o intenso nível de ruído em vários pontos da fábrica. As medições realizadas durante este trabalho são mostradas na Tabela 3.

Pode-se observar que o nível de ruído, ou supera o limite permitido pela Legislação Brasileira, $85 \mathrm{~dB}$, ou se situa em valores muito próximos deste limite.

\section{Avaliação técnica}

Os resultados das avaliações técnicas (inspeções) realizadas nesta fábrica, demonstram que a empresa possui um parque tecnológico obsoleto e poluidor, com altos níveis de contaminação individual que se reflete em casos de pneumoconioses, dermatites de contato e irritações diversas das vias aéreas superiores, altos índices de incidentes críticos e acidentes leves, ainda que subnotificados. O serviço médico não atende à demanda dos exames e a in-

\begin{tabular}{|c|c|c|c|}
\hline \multicolumn{4}{|c|}{$\begin{array}{l}\text { Resultados da avaliação individual de trabalhadores expostos à poeira, por função, } \\
\text { de ensacamento em uma empresa de cimento. Volta Redonda, Rio de Janeiro. }\end{array}$} \\
\hline Amostra* & Função & $\begin{array}{l}\text { Horário inicial } \\
\text { e final (horas) }\end{array}$ & $\begin{array}{l}\text { Concentração de } \\
\text { partículas }\left(\mathrm{mg} / \mathrm{m}^{3}\right)\end{array}$ \\
\hline ECC1 & Ensacador & $12: 22-16: 23$ & 41,87 \\
\hline ECC2 & Ensacador & $12: 20-16: 24$ & 52,44 \\
\hline $\mathrm{ECC} 3 * \star$ & Assistente & $12: 22-16: 26$ & 04,61 \\
\hline ECC4 & Ensacador & $13: 00-16: 37$ & 09,95 \\
\hline ECC5 & Ensacador & $13: 00-16: 38$ & 11,63 \\
\hline $\mathrm{ECC} 6 * \star \star$ & Carregador & $13: 00-16: 15$ & 03,59 \\
\hline
\end{tabular}

* Setor de Ensacamento, amostra por Ensacador de Cimento e Carregador (ECC). ** Controla o painel e a reposição de embalagens vazias.

$\star \star \star$ Carrega o caminhão com sacos de cimento cheios.

Tabela 2

Resultados da avaliação ambiental da concentração de poeira, nos setores de ensacamento fechado e aberto e no carregamento de caminhão de uma fábrica de cimento. Volta Redonda, Rio de Janeiro.

\begin{tabular}{|c|c|c|c|c|c|}
\hline Setor & Tempo (minutos) & $\begin{array}{l}\text { Número de } \\
\text { pontos avaliados }\end{array}$ & $\begin{array}{l}\text { Valores extremos } \\
\text { da leitura (cpm) }\end{array}$ & $\begin{array}{l}\text { Concentração } \\
\text { de particulados } \\
\left(\mathrm{mg} / \mathrm{m}^{3}\right)\end{array}$ & Desvio padrão $(\sigma)$ \\
\hline Ensacamento fechado & 10 & 13 & 225 a 1.520 & 1,57 a 11,88 & 1,90 \\
\hline Ensacamento aberto & 10 & 09 & 21 a 3.433 & 0,11 a 24,34 & 6,42 \\
\hline Carregamento de caminhão & 10 & 03 & 41 a 1.520 & 0,24 a 10,85 & - \\
\hline
\end{tabular}

$\mathrm{cpm}=$ contagens por minuto 
Tabela 3

Resultados da avaliação ambiental do nível de ruído, em decibéis, em setores críticos de uma fábrica de cimento. Volta Redonda, Rio de Janeiro.

\begin{tabular}{lrl}
\hline Setor & LA máximo $(\mathrm{dB})$ & Posto avaliado \\
\hline Ensacamento fechado & 85 & Carregamento \\
Ensacamento aberto & 86 & Ensacadeira \\
Ensacamento fechado & 83 & Carregamento \\
Ensacamento aberto & 85 & Ensacadeira \\
Silo 4 & 95 & Controle \\
Moinho 4 & $84-95$ & Próximo ao compressor \\
Moinho 4 & 101 & Área interna \\
Moinho 5 & $88-90$ & Área externa \\
Moinho 5 & 110 & Área interna \\
\hline
\end{tabular}

vestigação clínica se restringe a parâmetros sintomatológicos, e não estabelece rotinas de acordo com o posto, a função ou os problemas ambientais presentes no setor de trabalho.

A gerência de riscos se limita à identificação de pontos grosseiros e recomendação de equipamento de proteção individual. Esta atitude é também incorporada pela Comissão Interna de Prevenção de Acidentes (CIPA), que acaba por se tornar uma reunião de cobranças mútuas, ao invés de momentos de reflexão conjunta.

A esses resultados, deve-se acrescentar as questões ligadas à organização do trabalho, como as situações de penosidade, o trabalho em turnos, a supervisão permanente, o trabalho repetitivo, a submissão à pressão do tempo e ritmo da máquina e o trabalho em altura. É reconhecido que estes fatores contribuem significativamente para um aumento do risco de acidentes, agridem o organismo por desrespeitar os ritmos biológicos, além de contribuirem para a desorganização na vida familiar e social do trabalhador, impossibilitando-o de um convívio tranqüilo e planejado na vida domiciliar.

\section{As impressões dos trabalhadores}

Todos os 66 trabalhadores entrevistados, que compreendem o universo amostral da terceira etapa da vigilância, eram do sexo masculino, predominantemente jovens. A maioria, 63\%, tem menos de quarenta anos. O grau de escolaridade é baixo, sendo que $44 \%$ possui o primeiro grau completo e $35 \%$ incompleto. Sessenta e cinco por cento dos trabalhadores começou a trabalhar antes dos 18 anos, o que remonta ao contato bastante precoce com a realidade industrial.
O trabalho é organizado em jornadas mistas de 8 horas diárias e em sistema de rodízio de seis horas diárias. A maioria dos trabalhadores $(66,7 \%)$, relatou trabalhar em jornadas superiores a oito horas diárias, especialmente o pessoal da produção, com destaque para $100 \%$ dos que trabalham nas ensacadeiras e $77 \%$ dos que atuam na manutenção.

Em relação à capacitação para o exercício das atividades, constatou-se que em $57,6 \%$ dos casos, o treinamento foi feito de maneira informal na própria empresa, e uma pequena parcela $(21,1 \%)$, pelas instâncias de formação patronal, como por exemplo, o SENAI (Serviço Nacional de Aprendizagem Industrial). Esta precariedade do processo de capacitação, exerce um papel fundamental na ocorrência de situações de quase-acidentes, pois $35 \%$ dos trabalhadores relataram já ter se acidentado e $22,4 \%$ já foram atores em situações de quaseacidentes ou incidentes críticos.

A precária formação, tanto no que tange à capacitação para o exercício da função quanto na informação dos riscos decorrentes do trabalho, aliada à atuação punitiva da (CIPA) e à distância, do movimento sindical ou de qualquer outra forma de organização, redundam em estratégias de sobrevivência isoladas. Decorre desta conjunção de situações, a redução na leitura dos acidentes, responsabilizando o trabalhador. Leitura muitas vezes consensual, absorvida pelo próprio trabalhador e refletida na atribuição das causas correntes dos acidentes:

- erro humano (40\%);

- falta de manutenção e conservação de equipamentos (40\%);

- falta de política de segurança da empresa (10\%);

- uso de equipamento de proteção individual (EPI) inadequado (10\%).

As condições de trabalho, num parque industrial antigo e, em alguns casos obsoleto, foi bastante criticada pelos trabalhadores, que consideraram as máquinas de má qualidade em $21,4 \%$ das respostas, barulhentas para $55,46 \%$ e sem manutenção para $21,4 \%$.

A Tabela 4, descreve os fatores mais relatados como incômodos para os trabalhadores em seus ambientes de trabalho.

Em setores críticos como nas ensacadeiras e nos moinhos, o ruído, o calor, o ritmo e a poeira foram apontados por todos como fatores geradores de incômodo. Estas respostas estão de acordo com os níveis medidos nas avaliações ambientais.

A Tabela 5, mostra as queixas mais comuns de problemas de saúde nos diversos setores desta fábrica. A forte concordância entre as 
queixas relacionadas e a exposição ao ruído a que os trabalhadores são submetidos, por períodos freqüentemente superiores a 8 horas diárias, não têm sido registrados ou mesmo percebidos pelo serviço médico da empresa. Os exames periódicos e demissionais dos trabalhadores em atividade, não registram quaisquer investigação diagnóstica relacionada aos riscos; explica-se esta ausência de nexo pela não realização de exames específicos no conjunto de procedimentos periódicos, admissionais ou demissionais, como exames audiométricos, dermatológicos ou neurológicos. Mesmo o raio $\mathrm{X}$ de tórax, elementar na avaliação individual de trabalhadores expostos continuamente à poeira, não se mostrou de boa qualidade nem atualizados.

Dentre as áreas consideradas mais perigosas pelos trabalhadores, destacam-se a ponte rolante $(20 \%)$, os moinhos $(20 \%)$, os silos (11\%) e as ensacadeiras (8\%).

Constata-se, em claro acordo com a avaliação ambiental realizada, que os trabalhadores desses setores têm plena consciência dos riscos aos quais estão expostos, o que pode ser evidenciado também, quando se compara as queixas de problemas de saúde dos mesmos com os fatores que os incomodam em seu ambiente de trabalho.

\section{Conclusões}

A ação de vigilância pluriinstitucional e interdisciplinar, acompanhada do sindicato e subsidiada pelo olhar dos trabalhadores sobre suas condições ambientais, propiciou a identificação de questões sutis e muito abrangentes, inviável por métodos de avaliação isolados.

Pelos resultados obtidos neste trabalho, fica patente que o ambiente e o processo de trabalho são insalubres e podem colocar em risco a saúde dos trabalhadores. A tecnologia utilizada na fábrica é obsoleta e contribui para a alta concentração de poeiras minerais e para o elevado nível de ruído, observados nos ambientes de trabalho. Estes parâmetros por si só, nos níveis observados, já são capazes de causar danos à saúde dos trabalhadores. Entretanto, outros fatores de risco foram também identificados, contribuindo para uma maior insalubridade, o que faz com que seja necessária a adoção de ações imediatas por parte dos empresários para uma solução definitiva destes problemas.

Revela-se importante a mudança no papel da CIPA dessa fábrica, que de equipe punitiva, deveria passar a assessora e orientadora dos trabalhadores e da empresa, no que tange às questões de saúde no trabalho.

Algumas alterações nos procedimentos da vigilância estão em curso nesta empresa, e delas constam o aprimoramento do diagnóstico clínico e radiológico e a implementação das modificações sugeridas pela SES, que consiste na reorganização ampliada da área industrial, a partir da substituição completa do maquiná-

\begin{tabular}{|c|c|}
\hline \multicolumn{2}{|c|}{$\begin{array}{l}\text { Freqüência percentual de situações que causam incômodos, segundo } \\
\text { os trabalhadores de uma fábrica de cimento. Volta Redonda, Rio de Janeiro. }\end{array}$} \\
\hline Situações geradoras de incômodo & Freqüência percentual \\
\hline Calor & 24,4 \\
\hline Ruído & 21,2 \\
\hline Ritmo de trabalho & 13,5 \\
\hline Posição de trabalho & 12,8 \\
\hline Iluminação & 11,5 \\
\hline Poeira & 9,0 \\
\hline Supervisão & 6,4 \\
\hline Esforço físico & 5,8 \\
\hline
\end{tabular}

Tabela 5

Freqüência percentual das queixas de saúde mais freqüentes por trabalhadores, segundo setores específicos de uma fábrica de cimento. Volta Redonda, Rio de Janeiro.

\begin{tabular}{|c|c|c|c|c|c|c|}
\hline \multirow[t]{2}{*}{ Setor } & \multicolumn{6}{|c|}{ Queixas de saúde, em freqüência percentual } \\
\hline & Irritação & Agressividade & Nervosismo & Cefaléia & Audição & Ansiedade \\
\hline Carregamento & 50 & 50 & 50 & 50 & - & - \\
\hline Ensacamento & 60 & 30 & 75 & 75 & 30 & 50 \\
\hline Operador de moinho & 50 & 70 & 20 & 35 & - & 50 \\
\hline Manutenção & 40 & 20 & 25 & 20 & 12 & 20 \\
\hline
\end{tabular}

Os setores de ensacamento e de manutenção apresentaram significância estatística para $p<0,005$. 
rio do setor de ensacadeira, a modernização da ponte rolante e dos moinhos.

$\mathrm{O}$ atendimento a este propósito pressupõe a adoção de um Termo de Compromisso, onde cabe à empresa a mudança dos aspectos organizacionais do trabalho, a modernização do parque tecnológico, uma completa reformulação na gerência de risco, adotando técnicas e conceitos mais modernos, abrangentes e eficazes acompanhado por uma comissão composta de trabalhadores, técnicos do Serviço de Engenharia e Segurança e de técnicos do poder público.

A etapa seguinte à assinatura do Termo, consiste no estabelecimento de um cronogra- ma de mudanças e na transformação paulatina da cultura de segurança, estabelecendo novas formas de diálogo entre os trabalhadores e a gerência industrial, reservando ao Estado uma atuação pontual em casos extremos.

Muitas etapas no processo de vigilância ainda carecem de suporte teórico. Questões como o sofrimento psíquico, as repercussões orgânicas resultantes de pressões conjugadas de riscos variados, os reflexos da subjetividade na percepção e na resposta individual a riscos, carecem de sistematização metodológica pela academia e estão completamente invisíveis aos Serviços de Saúde do Trabalhador.

\section{Referências}

ALVEAR-GALINDO, M. G.; MENDEZ-RAMIREZ, I.; VILLEGAS-RODRIGUES, J. A.; CHAPELA-MENDOZA, R.; ESLAVA-CAMPOS, C. A. \& LAURELL, A. C., 1999. Risk indicator of dust exposure and health effects in cement plant workers. Journal of Occupational and Environmental Medicine, 41: 654-661.

CESTEH (Centro de Estudos da Saúde do Trabalhador e Ecologia Humana), 2000. As Indústrias Cimenteiras de Cantagalo que Realizam Co-Processamento de Resíduos Industriais. Relatório Técnico. Rio de Janeiro: CESTEH, Escola Nacional de Saúde Pública, Fundação Oswaldo Cruz.

DEJOURS, C., 1994. Psicodinâmica do Trabalho. São Paulo: Atlas.

JAPAN ASSOCIATION FOR WORKING ENVIRONMENT MEASUREMENT, 1991. Working Environment Measurement System in Japan. 3rd Ed. Tokyo: Japan Association for Working Environmental.

JISHA (Japan Industrial Safety and Health Association), 1985. Industrial Hygienist Technical Course, 6:35-48.

KIMURA, K., 1978a. T-R Dust Sampler (Rep.1). Journal Science of Labour, 54:11-18.

KIMURA, K., 1978b. T-R Dust Sampler (Rep.2). Journal Science of Labour, 54:663-670.

LAURELL, C. \& NORIEGA, M., 1989. Processo de Produção e Saúde: Desgaste Operário. São Paulo: Editora Hucitec.

MENDES, R., 1995. Patologia do Trabalho. Rio de Janeiro: Atheneu.

MINISTÉRIO DO TRABALHO, 1996. Normas Regulamentadoras de Segurança e Saúde no Trabalho, NR-15 Relativa à Atividade e Operações Insalubres. Brasília: Ministério do Trabalho.

RIBEIRO, F. S. N., 1996. Vigilância como Prática de Cidadania ou de Tutela? Rio de Janeiro: Programa de Saúde do Trabalhador, Secretaria de Estado de Saúde do Rio de Janeiro. (mimeo).

SANTOS, O. F., 1997. A Vigilância em Saúde do Trabalhador nas Indústrias de Cimento de Volta Redon- $d a-R J$. Monografia do Curso de Especialização em Saúde do Trabalhador, Rio de Janeiro: Centro de Estudos da Saúde do Trabalhador e Ecologia Humana, Escola Nacional de Saúde Pública, Fundação Oswaldo Cruz.

SOTO, J. M. O. G.; SAAD, I. F. S. D. \& FANTAZZINI, M. L., 1990. Riscos Químicos. São Paulo: Fundação Jorge Duprat Figueiredo de Segurança e Medicina do Trabalho.

TAMBELLINI, A., 1988. Avanços na Formulação de uma Política Nacional de Saúde no Brasil: As Atividades Subordinadas à Área das Relações Produção e Saúde. Rio de Janeiro: Centro de Estudos da Saúde do Trabalhador e Ecologia Humana, Escola Nacional de Saúde Pública, Fundação Oswaldo Cruz. (mimeo).

VASCONCELLOS, L. C. F., 1994. Os Caminhos do Caminho - a Municipalização como Estratégia de Consolidação da Saúde do Trabalhador no Setor Saúde. Dissertação de Mestrado. Rio de Janeiro: Escola Nacional de Saúde Pública, Fundação Oswaldo Cruz.

VASCONCELLOS, L. C. F. \& RIBEIRO, F. S. N., 1997. Investigação epidemiológica e intervenção sanitária em Saúde do Trabalhador - O planejamento segundo bases operacionais. Cadernos de Saúde Pública, 13:269-275.

VESTBO, J. \& RASMUSSEN, F. V., 1990. Long-term exposure to cement dust and later hospitalization due to respiratory disease. International Archives of Occupational and Environmental Health, 62: 217-220.

YANG, C. Y.; HUANG, C. C.; CHIU, H. F.; CHIU, J. F.; LAN, S. J. \& KO, Y. C., 1996. Effects of occupational dust exposure on the respiratory health of Portland cement workers. Journal of Toxicology and Environmental Health, 49:581-588.

Recebido em 14 de dezembro de 1999

Versão final reapresentada em 20 de dezembro de 2001 Aprovado em 15 de fevereiro de 2002 